\title{
Qualidade sanitária de sementes de aveia preta cv. brs 139 (Avena strigosa SCHREB) submetidas ao envelhecimento acelerado
}

\author{
Sanitary Quality of Black Oat Seeds cv. Brs 139 (Avena strigosa SCHREB) submitted to accelerated aging \\ Marciele Barbieri, Viviane Schons de Ávila, Caciara Gonzatto Maciel, Gisele Noal, \\ Marlove Fátima Brião Muniz, Andrea Cristina Dörr \\ Universidade Federal de Santa Maria - UFSM
}

\section{Resumo}

O trabalho objetivou estudar os efeitos de diferentes tratamentos na sanidade de sementes de aveia preta cv. BRS 139. As sementes foram submetidas aos seguintes tratamentos: Vincit ${ }^{\circledR} 50 \mathrm{SC}$, Vincit ${ }^{\circledR} 50 \mathrm{SC}+$ Trichodel $^{\circledR}$, Trichodel ${ }^{\circledR}$ e Testemunha sem tratamento. Em seguinda, foram acondicionadas, em caixas do tipo gerbox, com substrato papel-filtro umedecido em água destilada e esterilizada e permaneceram em câmaras B.O.D. na temperatura de $25^{\circ} \mathrm{C}$, com fotoperíodo de 12 horas, até a avaliação. Os fungos associados às sementes foram identificados com o auxílio de microscópios estereoscópico e óptico, após sete dias de incubação. O delineamento estatístico utilizado foi inteiramente casualizado com quatro tratamentos e oito repetições de 50 sementes cada. Os fungos Aspergillus spp, Fusarium spp., Penicillium spp., Rhizoctonia spp., Rhizopus spp. e Trichoderma spp. foram identificados nas sementes. Os resultados mostraram que o produto químico (Vincit ${ }^{\circledR} 50 \mathrm{SC}$ ), isolado ou associado com Trichodel ${ }^{\circledR}$, foi eficaz para o controle de fungos potencialmente patogênicos para as sementes de aveia preta. Conclui-se que os tratamentos mais eficientes para o controle dos fungos presentes consistem no produto químico Vincit ${ }^{\circledR} 50 \mathrm{SC}$ utilizado isoladamente e quando associado ao produto biológico Trichodel ${ }^{\circledR}$.

Palavras-chave: Aveia preta, fungos; Trichodel ${ }^{\circledR}$; Vincit ${ }^{\circledR} 50$ SC, envelhecimento acelerado.

\begin{abstract}
The work aimed to study the effects of different treatments on seed health black oat cv. BRS 139. The seeds were subjected to the following treatments: Vincit ${ }^{\circledR} 50 \mathrm{SC}$, Vincit ${ }^{\circledR}$ Trichode $^{\circledR}+50 \mathrm{SC}$, Trichode $\mathrm{l}^{\circledR}$ and Witness without treatment. Then, were packed in boxes of seed dispersal, substrate type filter paper dampened with distilled water and sterile and remained in chambers at temperature of B.O.D $25^{\circ} \mathrm{C}$, with photoperiod of 12 hours, until the assessment. Fungi associated with the seeds were identified with the aid of stereoscopic microscopes and optical, after seven days of incubation. The statistical design was a completely randomized with four treatments and eight reps of 50 seeds each. The fungi Aspergillus spp., Fusarium spp., Penicillium spp., Rhizoctonia spp., Rhizopus spp. and Trichoderma spp. were identified in seeds. The results showed that the chemical (Vincit ${ }^{\circledR} 50 \mathrm{SC}$ ), isolated or associated with Trichodel ${ }^{\circledR}$, was effective for the control of potentially pathogenic fungi to Oat seeds black. It is concluded that the most efficient treatments for the control of fungi present consist in chemical Vincit $^{\circledR} 50$ SC used alone and when associated with the biological product Trichodel ${ }^{\circledR}$.

Keywords: black Oats, fungi; Trichodel ${ }^{\circledR}$; Vincit ${ }^{\circledR} 50 \mathrm{SC}$, accelerated aging.
\end{abstract}




\section{INTRODUÇÃO}

A aveia preta (Avena strigosa SCHREB.) é uma gramínea pertencente à família das Poaceae, utilizada como planta forrageira e também para a produção de grãos. Segundo Bevilaqua e Pierobom (1995), essa forrageira de inverno, juntamente com o azevém, apresentam grande relevância econômica. O uso de sementes de alta qualidade é um dos fatores principais na implantação e no estabelecimento da lavoura no campo. Soave e Wetzel (1987) argumentam que alguns fungos patogênicos transmitidos pelas sementes são economicamente importantes, pois constituem fontes de inóculo para o desenvolvimento de doenças e podem interferir na emergência e estabelecimento das culturas.

$\mathrm{O}$ vigor das sementes afeta diretamente a emergência das plântulas e o estabelecimento das culturas no campo, podendo gerar perdas na produção. Estes aspectos de desempenho incluem a taxa e uniformidade de germinação de sementes e crescimento de plântulas em laboratório (SCHUCH et al., 1999), a emergência e crescimento de plântulas no campo (SCHUCH e LIN, 1982; SCHUCH, 1999), a habilidade de emergência de plântulas em condições ambientais desfavoráveis, além de afetar o crescimento e rendimento da planta. O mesmo autor relata que, quanto ao teste de envelhecimento acelerado tradicional (com água), as diferenças na absorção de água pelas sementes, expostas à atmosfera com alta umidade relativa podem originar variações acentuadas no grau de umidade das sementes. Além disso, as sementes com alto vigor mantem sua capacidade de produzir plântulas normais e apresentam germinação mais elevada após serem submetidas a tratamentos de envelhecimento acelerado, enquanto as menos vigorosas apresentam maior redução de sua viabilidade.

O envelhecimento acelerado tradicional tem sido realizado em sementes de aveia preta a $42{ }^{\circ} \mathrm{C}$ por 48 horas (NAKAGAWA et al., 1994; NAKAGAWA et al., 1995; PATERNIANI et al., 1995). Já Garcia e Menezes (1999), quando estudaram a metodologia do teste, testaram uma única temperatura $\left(41^{\circ} \mathrm{C}\right)$ e verificaram que 24 horas foi o melhor período de condicionamento. A metodologia, dessa forma, precisa ser aprimorada para avaliação do vigor das sementes dessa cultura, em relação ao teste de envelhecimento acelerado.

Segundo Tanaka (1982), mesmo utilizando sementes sadias, muitas doenças podem ocorrer no campo como, por exemplo, por contaminação e pelos processos naturais de disseminação dos patógenos. Além disso, as sementes podem cons- tituir um veículo de disseminação de patógenos para áreas livres. Neste sentido, as sementes são responsáveis por propagar, aproximadamente, 90\% das plantas cultivadas destinadas à alimentação humana e animal (NEERGAARD, 1979). Segundo Machado (1988), entre os agentes patogênicos para plantas, os fungos são os mais ativos, tendo uma maior habilidade em penetrar diretamente nos tecidos vegetais e se alojarem mais facilmente. O inóculo pode ser transportado via semente, na forma de micélio e/ou de esporos, mas a taxa de transmissão do patógeno, entre outros fatores, depende fundamentalmente da quantidade e localização do inóculo na semente (NEERGAARD, 1979; MACHADO, 1988; MENTEN, 1991a).

Para a melhoria da qualidade fisiológica e sanitária das sementes de cereais de inverno, o emprego de fungicidas eficientes tem por objetivo principal reduzir, em baixos níveis, a taxa de transmissão dos patógenos, visando secundariamente, a elevação da emergência (FORCELINI, 1995). Segundo Henning et al. (2009) os fungicidas biológicos e alguns formulados com isolados de Trichoderma spp., apresentam amplitude de ação no antagonismo a fungos e bactérias. Esses microrganismos são atóxicos ao homem e aos animais. Além dos efeitos de Trichoderma spp. no controle de patógenos de plantas, determinadas linhagens podem ter efeito estimulatório no crescimento e no florescimento de plantas hortícolas.

Além disso, para o controle de patógenos utiliza-se o tratamento químico, o qual tem sido uma alternativa crescente em termos de adoção. De acordo com Machado et al. (2006), o controle químico consiste na eficácia e rapidez, impedindo a competição com plantas daninhas a partir da instalação da cultura na lavoura, permitindo assim o controle de invasoras.

Diante do exposto, o objetivo do trabalho consiste em estudar a eficiência de sementes de aveia preta das cultivares BRS 139 submetidas a diferentes tratamentos em relação à qualidade sanitária quando conduzidas ao envelhecimento acelerado.

\section{METODOLOGIA}

\section{I Aquisição das sementes e recepção no laboratório}

As sementes de aveia preta cv. BRS 139 e aveia preta cv. EMBRAPA 139 foram produzidas no município de Júlio de Castilhos-RS, e obtidas 
através de um produtor local. Os trabalhos foram desenvolvidos no Laboratório de Fitopatologia Elocy Minussi do Departamento de Defesa Fitossanitária da Universidade Federal de Santa Maria durante o período de março a outubro de 2012, e em área experimental neste mesmo município, também neste mesmo intervalo de tempo. Após o recebimento, as sementes de aveia preta foram submetidas aos diferentes tratamentos e ao envelhecimento acelerado.

\subsection{Submissão das sementes ao tratamento químico e biológico.}

O tratamento químico utilizado para o controle com fungicida foi Vincit ${ }^{\circledR}$ 50SC (fungicida sistêmico do grupo químico triazol, suspensão concentrada (SC) e indicado para uso em tratamento de sementes nas culturas de algodão, aveia, cevada, feijão, soja e trigo), o qual é registrado no Ministério da Agricultura, Pecuária e Abastecimento - MAPA. Para a cultura da aveia este produto controla Helmintosporiose (Drechslera avenae) e Mancha-de-Alternaria (Alternaria alternata).

O tratamento biológico constituiu-se no uso de Trichodel ${ }^{\circledR}$, produto biológico composto de linhagens selecionadas do fungo Trichoderma spp. e que inibe o desenvolvimento de fungos patogênicos presentes no solo e sobre as plantas.

As sementes foram submetidas aos tratamentos: T1 (testemunha), T2 (Vincit ${ }^{\circledR}$ 50SC), T3 $\left(\right.$ Vincit $^{\circledR} 50 \mathrm{SC}+$ Trichodel $\left.^{\circledR}\right)$ e T4 $\left(\right.$ Trichodel $\left.^{\mathbb{}}\right)$ e as doses de cada produto foram seguidas conforme a indicação anexada a cada um desses produtos. Utilizou-se, para o produto Vincit ${ }^{\circledR} 50 \mathrm{SC}$, o recomendado $150 \mathrm{~mL}$ para $100 \mathrm{~kg}$ de aveia; então, 1,25 $\mathrm{kg}$ (quantidade utilizada para todo o experimento tanto a campo como em laboratório) de aveia resultando em 1,9 mL de Vincit ${ }^{\circledR} 50 S C$. Da mesma maneira, conduziu-se o tratamento das sementes com o produto biológico, ou seja, o recomendado para o produto Trichodel ${ }^{\circledR}$ é de $250 \mathrm{~mL} / \mathrm{saco}$ de $40 \mathrm{~kg}$ de aveia; então, 1,25 kg de aveia (quantidade utilizada para todo o experimento tanto a campo como em laboratório) resultando em $6,25 \mathrm{~mL}$ de Trichodel ${ }^{\circledR}$.

\subsection{Submissão das sementes ao estresse pelo envelhecimento acelerado}

Após os tratamentos, as sementes foram expostas a uma temperatura que oscilou entre 40 e $43^{\circ} \mathrm{C}$, em diferentes tempos de exposição: 0 - 24 - 48 e 72 horas. Posteriormente, submeteram-se as sementes aos testes de avaliação de sanidade. $\mathrm{O}$ teste de sanidade foi realizado com oito repetições de 50 sementes que foram colocadas em caixas do tipo gerbox previamente desinfetadas com solução de hipoclorito de sódio (1\%) e álcool (70\%). Utilizaram-se como substrato duas folhas de papel-filtro, umedecidas com água destilada esterilizada. Adicionou-se $40 \mathrm{ml}$ de água e as sementes foram dispostas em uma tela do formato da gerbox, posicionadas na parte superior de forma que não entre em contato com água. A verificação da ocorrência de fungos foi feita com auxílio de microscópio estereoscópico, no qual as sementes foram observadas individualmente, através do microscópio óptico e literatura disponível (BARNETT e HUNTER, 1999) quanto a identificação dos fungos em nível de gênero.

Para os testes de germinação, as sementes foram colocadas sobre duas folhas de papel-filtro estéril, umedecido com água destilada, em um número de 25 sementes por repetição, em um total de oito repetições. A primeira contagem foi realizada cinco dias após a instalação do teste, enquanto a contagem final foi feita aos 10 dias, conforme metodologia descrita por Brasil (2009). Analisou-se o vigor, na primeira contagem, e germinação total através do somatório entre a primeira e segunda contagem. Os percentuais quando somados resultaram no potencial germinativo das sementes; outras variáveis consistiram em plântulas anormais e sementes mortas. A partir das plântulas vigorosas fez-se a medição do comprimento da parte aérea e radicular, para isso foram selecionadas aleatoriamente 10 plântulas normais de cada repetição.

\section{RESULTADOS E DISCUSSÃO}

Para a cultivar BRS 139, verificou-se a presença dos seguintes fungos associados às sementes: Rhizoctonia spp., Rhizopus spp., Aspergillus spp., Penicillium spp., Fusarium spp. e Trichoderma spp. (Figura 1). A associação patógeno semente consiste em uma das maneiras mais beneficentes para a sobrevivência e a disseminação destes agentes, pois quando se refere às sementes, não há presença de barreiras (NEERGARD, 1977; TANAKA, 1982; MACHADO, 1988 e WETZEL, 1988). Esta associação, portanto, é importante pelo fato de que o patógeno sobrevive por mais tempo, mantendo sua viabilidade e características. Consiste também na facilidade de disseminação e com isso, pode ser introduzido em novas áreas e com alta 
probabilidade do patógeno infectar a plântula em crescimento posteriormente à semeadura, provocando severas doenças na fase inicial da cultura (BAKER, 1972 e MENTEN, 1995).

A incidência de Rhizoctonia spp. em sementes de aveia preta da cultivar BRS 139 é praticamente inexistente. Na figura $1 \mathrm{~A}$, pode-se verificar que há uma mínima percentagem de sua existência na testemunha (T1) dentro da faixa do tempo zero ao de 24 horas; onde posteriormente não se nota a presença de Rhizoctonia spp.. Isso se deve ao eficiente controle através dos tratamentos utilizados, quando essas sementes foram submetidas ao envelhecimento acelerado sob estresse elevado de temperatura e umidade.

A presença de Rhizopus spp., na figura 1B, foi crescente entre o tempo zero e o de 24 horas. Observa-se que em seguida diminui quando as sementes de aveia preta cv. BRS 139 foram expostas a elevadas temperaturas por um período de tempo maior, consequentemente houve maior deteriorização. Conforme Balardin e Loch (1987), os fungos de armazenamento dos gêneros Aspergillus spp., Penicillium spp., Cladosporium spp., Epicoccum spp., Curvularia spp. e Rhizopus spp.. foram constatados de forma geral nos lotes estudados de aveia preta, em diferentes proporções. Este resultado pode ocasionar prejuízos na qualidade fisiológica das sementes, como também, comprometer o estande inicial da lavoura.

Assim, observa-se que o tratamento das sementes com o produto biológico Trichodel ${ }^{\circledR}$ não foi eficiente no controle de Rhizopus spp.. Da mesma forma, Milanesi et al. (2006), destaca que nas sementes de aveia tratadas com Agrotrich ${ }^{\circledR}$, houve um aumento na incidência de Rhizopus spp., Aspergillus niger e Penicillium spp., demonstrando que o bioprotetor não apresentou um controle efetivo em relação à testemunha.

Em relação à Aspergillus spp. (Figura 1C) no tempo zero, verificou-se que houve maior incidência. Este fungo tem desenvolvimento crescente conforme o aumento do tempo de exposição a altas temperaturas e maior período de armazenamento, semelhante ao que ocorreu quando as sementes de aveia preta foram tratadas com Trichodel ${ }^{\circledR}$, para o fungo Aspergillus spp., demonstrando elevada variação de concentração do mesmo entre os tempos de 24 a 72 horas. Portanto, os tratamentos mais eficientes para o controle desse fungo consistem no produto químico Vincit ${ }^{\circledR}$ 50SC utilizado isoladamente e quando associado ao produto biológico Trichodel $^{\circledR}$.
Ao analisar a incidência de Penicillium spp. (Figura 1D) percebeu-se maior incidência entre o período de zero à 24 horas de exposição a temperatura e umidade elevada, e à medida que aumentou o tempo da exposição das sementes a essas condições houve decréscimo no desenvolvimento. Isso também foi percebido por Tanaka et al. (2001), os quais afirmam que o teor de umidade mais elevado favoreceu a incidência dos fungos dos gêneros Aspergillus spp., Penicillium spp. e Fusarium spp. e a incidência fúngica no início do armazenamento refere-se basicamente à contaminação vinda da lavoura, ou seja, fungos de campo, pois esses fungos desenvolvem-se melhor em umidades relativas mais elevadas. Além disso, como demonstrado para Aspergillus spp., os tratamentos são mais eficientes e representados pelo produto químico Vincit ${ }^{\circledR}$ 50SC utilizado isoladamente e quando associado ao produto biológico Trichodel ${ }^{\circledR}$.

Ao contrário desses fungos, o Trichoderma spp. (Figura 1E) obteve menor percentual de desenvolvimento. Observou-se que quando analisada sua presença que não houve diferença significativa entre os tratamentos, porém a associação do produto biológico com o produto químico não inibiu a colonização do Trichodel ${ }^{\circledR}$. Segundo Ethur et al. (2006), os tratamentos Rhodiauram $^{\circledR} S C$ e Rhodiauram ${ }^{\circledR} S C(2 \mathrm{~h})+$ Agrotrich $^{\circledR}$, reduziram $100 \%$ a contaminação por fungos nas sementes de aveia preta e também, nas sementes tratadas com Rhodiauram ${ }^{\circledR} \mathrm{SC}(2 \mathrm{~h})+$ Agrotrich $^{\circledR}$, foi observado que $3 \%$ apresentavam Trichoderma spp., demonstrando a capacidade do bioprotetor em colonizar mesmo na presença do fungicida.

Para o fungo Fusarium spp. (Figura 1F) também não houve diferença significativa entre os tratamentos utilizados. Contudo, percebeu-se que o produto biológico Trichodel ${ }^{\circledR}$ não foi inibido pelo produto químico Vincit ${ }^{\circledR} 50 \mathrm{SC}$. Assim, os tratamentos 2, 3 e 4 foram eficientes para o controle desse fungo. Neergard (1979) afirma que o tratamento com fungicidas é importante para controlar patógenos presentes nas sementes, conferir proteção nos casos de semeaduras profundas e possibilitar o escape do ataque de patógenos do gênero Fusarium spp. 


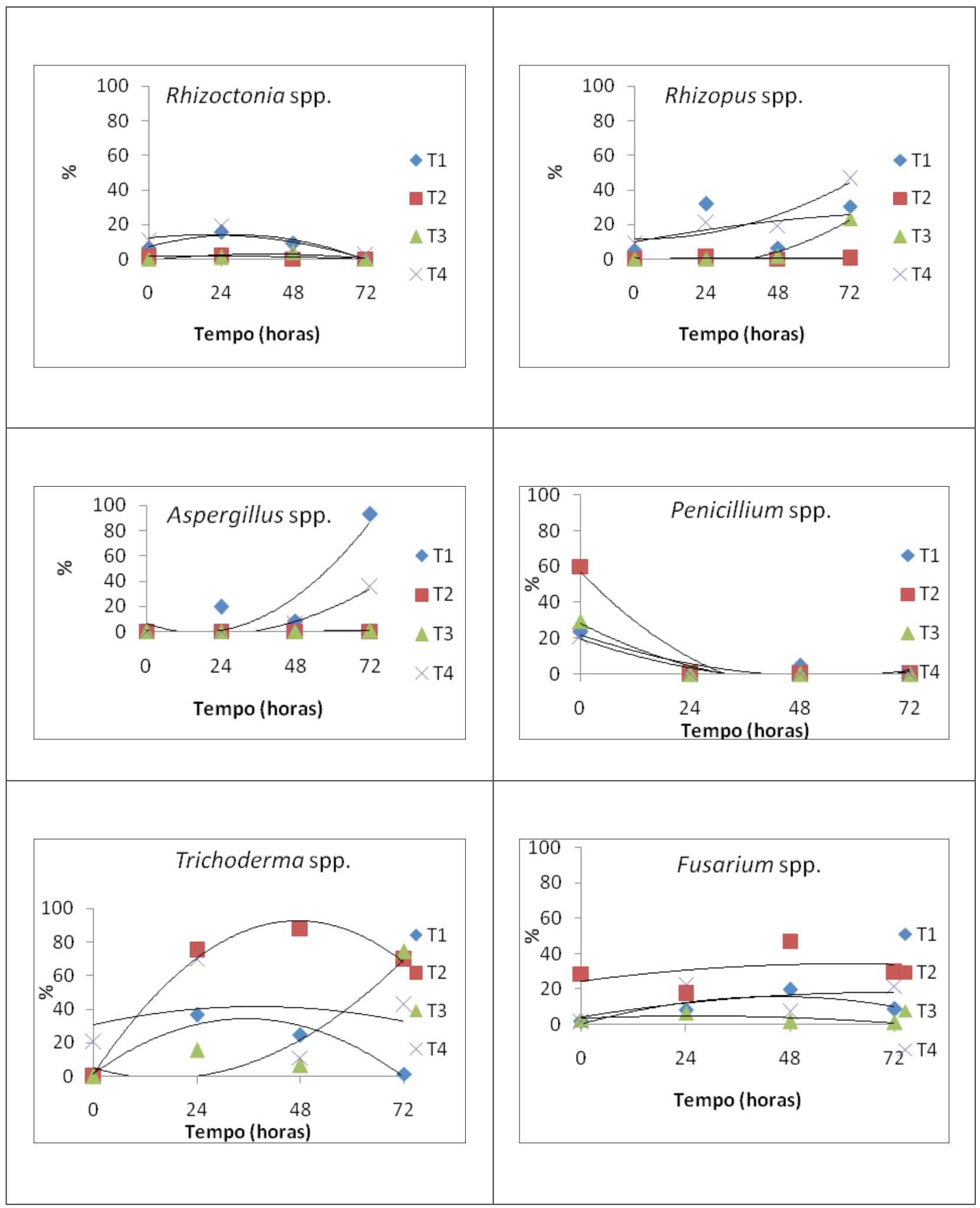

Figura 1 - Fungos associados às sementes de Avena strigosa cv. EMBRAPA 139 submetidas a diferentes tratamentos e ao envelhecimento acelerado. T1 - Testemunha; B - T2 - $\left(\right.$ Vincit $\left.^{\circledR} 50 \mathrm{SC}\right)$; T3 - $\left(\right.$ Vincit $^{\mathbb{}} 50 \mathrm{SC}+$ Trichodel $\left.^{\circledR}\right)$ e T4 - $\left(\right.$ Trichodel $\left.^{\circledR}\right)$.

Fonte: BARBIERI, M., Santa Maria, 2012. 
Tabela 1 - Equações das análises de regressão dos respectivos fungos associados às sementes de Avena strigosa cv. EMBRAPA 139 submetidas a diferentes tratamentos pelo ao envelhecimento acelerado.

\begin{tabular}{|c|c|c|c|}
\hline Patógeno & Tratamentos & Equação das análises de regressão & $\begin{array}{l}\text { Nível de } \\
\text { significância }\end{array}$ \\
\hline Rhizoctonia ssp. & $\begin{array}{l}\text { Testemunha } \\
\text { Vincit }^{\circledR} 50 \mathrm{SC} \\
\text { Vincit }^{\circledR} 50 \mathrm{SC}+\text { Trichodel }^{\circledR} \\
\text { Trichodel }^{\circledR}\end{array}$ & $\begin{array}{l}y=-0,008 x^{2}+0,471 x+7,075 \\
y=-0,000 x^{2}+0,024 x+1,325 \\
y=-0,002 x^{2}+0,168 x-0,45 \\
y=-0,005 x^{2}+0,190 x+12,32\end{array}$ & $\begin{array}{l}\mathrm{R}^{2}=0,947 \\
\mathrm{R}^{2}=0,495 \\
\mathrm{R}^{2}=0,623 \\
\mathrm{R}^{2}=0,633\end{array}$ \\
\hline Rhizopus spp. & $\begin{array}{l}\text { Testemunha } \\
\text { Vincit }^{\circledR} 50 \mathrm{SC} \\
\text { Vincit }^{\circledR} 50 \mathrm{SC}+\text { Trichodel }^{\circledR} \\
\text { Trichodel }^{\circledR}\end{array}$ & $\begin{array}{l}y=-0,001 x^{2}+0,328 x+9,625 \\
y=-0,000 x^{2}+0,017 x+0,175 \\
y=0,009 x^{2}-0,387 x+0,95 \\
y=0,006 x^{2}-0,028 x+11,57\end{array}$ & $\begin{array}{l}\mathrm{R}^{2}=0,211 \\
\mathrm{R}^{2}=0,109 \\
\mathrm{R}^{2}=0,954 \\
\mathrm{R}^{2}=0,884\end{array}$ \\
\hline Aspergillus spp. & $\begin{array}{l}\text { Testemunha } \\
\text { Vincit }^{\circledR} 50 S C \\
\text { Vincit }^{\circledR} 50 S C+\text { Trichodel }^{\circledR} \\
\text { Trichodel }^{\circledR}\end{array}$ & $\begin{array}{l}y=0,028 x^{2}-0,914 x+6,3 \\
y=0 \\
y=0,000 x^{2}-0,009 x+0,025 \\
y=0,012 x^{2}-0,461 x+0,925\end{array}$ & $\begin{array}{l}\mathrm{R}^{2}=0,854 \\
\mathrm{R}^{2}=0 \\
\mathrm{R}^{2}=0,933 \\
\mathrm{R}^{2}=0,979\end{array}$ \\
\hline Penicillium spp. & $\begin{array}{l}\text { Testemunha } \\
\text { Vincit }^{\circledR} 50 S C \\
\text { Vincit }^{\circledR} 50 S C+\text { Trichodel }^{\circledR} \\
\text { Trichodel }^{\circledR}\end{array}$ & $\begin{array}{l}y=0,008 x^{2}-0,868 x+21,65 \\
y=0,026 x^{2}-2,625 x+57 \\
y=0,012 x^{2}-1,290 x+28,02 \\
y=0,008 x^{2}-0,896 x+19,47\end{array}$ & $\begin{array}{l}\mathrm{R}^{2}=0,818 \\
\mathrm{R}^{2}=0,933 \\
\mathrm{R}^{2}=0,933 \\
\mathrm{R}^{2}=0,933\end{array}$ \\
\hline Trichoderma spp. & $\begin{array}{l}\text { Testemunha } \\
\text { Vincit }^{\circledR} 50 \mathrm{SC} \\
\text { Vincit }^{\circledR} 50 \mathrm{SC}+\text { Trichodel }^{\circledR} \\
\text { Trichodel }^{\circledR}\end{array}$ & $\begin{array}{l}y=-0,025 x^{2}+1,828 x+1,875 \\
y=-0,040 x^{2}+3,844 x+1,525 \\
y=0,022 x^{2}-0,746 x+5,075 \\
y=-0,007 x^{2}+0,591 x+30,45\end{array}$ & $\begin{array}{l}\mathrm{R}^{2}=0,926 \\
\mathrm{R}^{2}=0,990 \\
\mathrm{R}^{2}=0,853 \\
\mathrm{R}^{2}=0,040\end{array}$ \\
\hline Fusarium spp. & $\begin{array}{l}\text { Testemunha } \\
\text { Vincit }^{\circledR} 50 S C \\
\text { Vincit }^{\circledR} 50 S C+\text { Trichodel }^{\circledR} \\
\text { Trichodel }^{\circledR}\end{array}$ & $\begin{array}{l}y=-0,007 x^{2}+0,682 x+0,125 \\
y=-0,002 x^{2}+0,335 x+24,11 \\
y=-0,002 x^{2}+0,101 x+3,175 \\
y=-0,002 x^{2}+0,396 x+4,275\end{array}$ & $\begin{array}{l}\mathrm{R}^{2}=0,773 \\
\mathrm{R}^{2}=0,141 \\
\mathrm{R}^{2}=0,512 \\
\mathrm{R}^{2}=0,356\end{array}$ \\
\hline
\end{tabular}

Fonte: BARBIERI, M., Santa Maria, 2012. 


\section{CONCLUSÕES}

Conclui-se que os tratamentos mais eficientes para o controle dos fungos presentes consistem no produto químico Vincit ${ }^{\circledR} 50 \mathrm{SC}$ utilizado isoladamente e quando associado ao produto biológico Trichodel ${ }^{\circledR}$.

\section{REFERÊNCIAS}

BARNETT, H. L.; HUNTER, B. B. Illustred genera of imperfect fungi. $3 \mathrm{Ed}$. Minnesota: Burgess Publishing Company, 1999, p. 241.

BALARDIN, R.S.; LOCH, L.C. Efeito de thiram sobre a germinação de sementes de centeio e aveia. Revista Brasileira de Sementes, v.9, n.1, p.113-117, 1987.

BAKER, K. Seed pathology. In: KOZLOWSKI, T.(ed.). Seed biology. New York: Academic Press, 1972. v.2, p.317-416.

BARBOSA, M.A.G.et al. Antagonism of Trichoderma species on Cladosporum herbarum and their enzymatic characterization. Brazilian Journal of Microbiology, v.32, p.98-104, 2001.

BASRA, A. S. Seed quality - Basic mechanisms and agricultural implications. Foods Products Press, 1994. $389 \mathrm{p}$.

BRASIL, Ministério da Agricultura e Reforma Agrária. Regras para análises de sementes. Brasília, DF: SNAD/CLAV, 1992. 365p.

BEVILAQUA, G.A.; PIEROBOM, C.R. Qualidade sanitária e fisiológica de sementes de aveia-preta (Avena strigosa Schreb) da zona sul do Rio Grande do Sul. Revista Brasileira de Sementes, v.17, n.1, p.1922, 1995.

CARVALHO, N. M. Vigor de sementes. In: CÍCERO, S. M.; MARCOS FILHO, J. \& SILVA, W. R. (Coord.). Atualização em produção de sementes. Campinas: Fundação Cargill, 1986. p. 207-223.

COMISSÃO BRASILEIRA DE PESQUISA DE AVEIA. In: Indicações técnicas para cultura aveia. Guarapuava: A Comissão: Fundação Agrária de Pesquisa Agropecuária, 2006.
CORRÊA, E.B.; BETTIOL, W. Controle Biológico da Podridão de Raízes Causada por Pythium spp. em Cultivos Hidropônicos. Jaguariúna: Embrapa Meio Ambiente, 2009, pg. 26 - (Embrapa Meio Ambiente. Documentos; 77). .

DIAS, M.C.L.L.; BARROS, A.S.R. Avaliação da qualidade de sementes de milho. Londrina: IAPAR, 1995. 43p. (Circular, 88).

DELOUCHE, J. Germinação, deterioração e vigor da semente. Seed News, Pelotas, n. 6, p.24-31, 2002.

ETHUR, L.Z. et al.. Diferentes produtos na sanidade e vigor de sementes de zínia (Zinnia elegans).In: III CONGRESSO BRASILEIRO DE AGROECOLOGIA, 2005. Florianópolis. Anais III Congresso Brasileiro de Agroecologia. Florianópolis, 2005.

ETHUR, L.Z.; et al. Sanidade de sementes e emergência de plântulas de nabo forrageiro, aveia preta e centeio submetidas a tratamentos com bioprotetor e fungicida. Ciência e Natura, UFSM, v. 28, n. 2, p. $17-27,2006$.

FARIA, A.Y.K.; ALBUQUERQUE, M.C.F.; NETO, D.C. Qualidade fisiológica de sementes de algodoeiro submetidas a tratamentos químico e biológico. Revista Brasileira de Sementes, v.25, n.1, p.121-127, 2003.

FARIAS, C.R.J. et al., Qualidade sanitária de sementes de aveia-preta (avena strigosa schreb.) produzidas no estado do rio grande do sul, safra 1999/2000.

Revista Brasileira de Sementes, v. 24, n 1, p.1-4, 2002.

FARIAS, C.R.J.; DEL PONTE, E.M.; LUCCA FILHO, O.A.; PIEROBOM, C.R. et al. Fungos causadores de helmintosporiose associados às sementes de aveia-preta (Avena strigosa, schreb.). Revista Brasileira Agrociência, Pelotas, v.11, n.1, p.57-61, 2005.

FERREIRA, D.F. SISVAR: um programa para análises estatísticas e ensino de estatística. Revista Symposium, v.6, p.36-41, 2008.

FORCELINI, C.A. Tratamento de sementes de trigo no Brasil. In: MENTEN, J.O.M. Patógenos em sementes: detecção, danos e controle químico. São Paulo: Ciba Agro, 1995. p. 247 - 264.

HARMAN, G.E. Myth and dogmas of biocontrol changes in perceptions derived from research on Tri- 
choderma harzianum T-22. Plant Disease,v.84, n.4, p. 377-393, 2000.

HENNING, F.A.;et al.. Qualidade fisiológica, sanitária e análise de isoenzimas de sementes de aveia-preta tratadas com diferentes fungicidas. Revista Brasileira de Sementes, v. 31, n. 3, p.063-069, 2009.

HENNING, A.A.; HENNING, F.A. Microbiolization of soybean seeds. In: XIX Seminário Pan Americano de Semillas, Assunção. Conferencias y Resumenes de Trabajos Presentados. Assunción: FELAS, 2004. p. 330.

HENNING, F. A. et al. Qualidade fisiológica, sanitária e análise de isoenzimas de sementes de aveia-preta tratadas com diferentes fungicidas. Revista Brasileira de Sementes, vol. 31, n 3, p.063-069, 2009.

LIMA e BORGES, E. E. de; RENA, A. B. Germinação de sementes. In: Sementes florestais tropicais. Brasília: ABRATES, 1993.

MACHADO, J.C.; et al. Tratamento de sementes no controle de fitopatógenos e pragas. Informe Agropecuário, Belo Horizonte, v.27, n.232, p.76-87, maio/ jun. 2006.

MACHADO, J.C. Patologia de sementes: fundamentos e aplicações. Brasília: ESAL/FAEPE, 1988. $107 \mathrm{p}$.

MACIEL, C.G,; et al. Germinação e Sanidade de Sementes de Ipê-amarelo (Tabebuia serratifolia), após Submissão a Diferentes Tratamentos. Revista Brasileira de Agroecologia, v.4, n.2, p. 952 - 955, 2009.

MALAVASI, M. de M. Germinação de sementes. In: Manual de análise de sementes florestais. Fundação Cargill, 1988. 100 p.

MARTINS-CORDER, M.P.P.; MELO, I.S. Influência de Trichoderma viride e T. koningii na emergência de plântulas e no vigor de mudas de berinjela. Revista Brasileira de Biologia, v.57, n.1, p.39-45, 1997.

MATTHEWS, S. Physiology of seed ageing. Outlook on Agriculture, Elmsford, v.14, n.2, p.8994, 1985.

MENEZES, N.L. et al. Teste de condutividade elétrica em sementes de aveia preta. Revista Brasileira de Sementes, vol. 29, n 2, p.138-142, 2007.
MARCOS FILHO, J. Teste de envelhecimento acelerado. In: KRZYZANOWSKI, F.C.; VIEIRA, R.D.; FRANÇA NETO, J.B. (Ed.) Vigor de sementes: conceitos e testes. Londrina: Abrates, 1999. p.3.13.24 .

MARCOS FILHO, J.; CÍCERO, S.M.; SILVA, W.R. Avaliação da qualidade das sementes. Piracicaba: FEALQ, 1987. 230p.

McDONALD, M.B.; GUPTA, I.J.; SCHMITTHENNER, A.F. Effect of storage fungi on seed vigour of soybean. Seed Science and Technology, Zurich, v.21, n.3, p.581-591, 1993.

McDONALD Jr., M.B. Assessment for seed quality. HortScience, v.15, n.6, p.784- 788, 1980.

MELLO, V.D.C.; TILLMANN, M.A.A. O teste de vigor em câmara de envelhecimento precoce. Revista Brasileira de Sementes, Brasília, v. 9, n. 2, p. 93-102, 1987.

MEHTA, Y.R. Ocorrência de Drechslera spp. em aveia branca no Estado do Paraná. Summa Phytopathologica, São Paulo, v. 25, n. 3, p. 265-267, 1999.

MENTEN, J.O.M. Prejuízos causados por patógenos associados às sementes. In: MENTEN, J.O.M. (ed.). Patógenos em sementes: detecção, danos e controle químico. São Paulo: Ciba Agro, 1995, p.115-136.

NEERGAARD, P. Seed pathology. London: The MacMillan Press, 1977. v.2, 1191p.

NEERGAARD, P. Seed Pathology. 2. ed. London, MacMillan Press, v.2, 1979.

PANOBIANCO, M.; MARCOS FILHO, J. Comparação entre métodos para avaliação da qualidade fisiológica de sementes de pimentão. Revista Brasileira de Sementes, v.20, p.306-310, 1998.

SCHUCH, L.O.B. e LIN, S.S. Efeito do envelhecimento rápido sobre o desempenho de sementes e de plantas de trigo. Pesquisa Agropecuária Brasileira, Brasília, v.17, n.8, p.1163-1170, 1982.

SCHUCH, L.O.B. Vigor das sementes e aspectos da fisiologia da produção em aveia-preta (Avena strigosa Schreb.). Pelotas: Universidade Federal de Pelotas, 1999. 127p. (Tese Doutorado). 
SCHUCH, L.O.B.; et al. Crescimento em laboratório de plântulas de aveia-preta (Avena strigosa Schreb.) em função do vigor das sementes. Revista Brasileira de Sementes, Brasília, v.21, n.1, p.229-234, 1999.

SOAVE, J.; WETZEL, M.M.V. Patologia de sementes. Campinas: Fundação Cargill, 1987. 480p.

SOUZA, S.A.; NAKAGAWA, J.; MACHADO, C.G. Teste de envelhecimento acelerado em sementes de aveia preta. Revista Brasileira de Sementes, v.31, n.2, p.155-163, 2009.

TORRES, S.B. Métodos para avaliação do potencial fisiológico de sementes de melão. 2002. 103f. Tese (Doutorado em Agronomia) - ESALQ/USP, Piracicaba.

ROSSETTO, C.A.V; et al. Efeito da disponibilidade hídrica do substrato, da qualidade fisiológica e do teor de água inicial das sementes de soja no processo de germinação. Scientia Agricola, Piracicaba, v.54, n. 1-2, p.97-105, Jan./Ago. 1997.

PAOLINELLI, G.P. e BRAGA, S.J. Alterações da qualidade de sementes de algodão armazenadas com dois níveis de vigor. Informativo ABRATES, Curitiba, v.7, n.1/2, p.168, 1997.

TANAKA, M.A.S. Importância da utilização de sementes sadias. Informe Agropecuário, Belo Horizonte. v.8, n.91, p.31-34. 1982.

WETZEL, M.M.V.S. Controle sanitário na importação de sementes. In: SIMPÓSIO BRASILEIRO DE PATOLOGIA DE SEMENTES, 3, Lavras, 1988. Resumos. Campinas: Fundação Cargill, 1988. p.4857. 